\title{
Prescription Software for Recovery and Rehabilitation Using Microsoft Kinect
}

\author{
Stephen Simmons and Rachel McCrindle \\ School of Systems Engineering \\ University of Reading \\ Reading, UK \\ s.r.simmons@student.reading.ac.uk, \\ r.j.mccrindle@ reading.ac.uk
}

\author{
Malcolm Sperrin and Andy Smith \\ Medical Physics and Clinical Engineering \\ Royal Berkshire NHS Foundation Trust \\ Reading, UK \\ malcolm.sperrin@ royalberkshire.nhs.uk, \\ andy.smith@ royalberkshire.nhs.uk
}

\begin{abstract}
Brain injuries, including stroke, can be debilitating incidents with potential for severe long term effects; many people stop making significant progress once leaving in-patient medical care and are unable to fully restore their quality of life when returning home. The aim of this collaborative project, between the Royal Berkshire NHS Foundation Trust and the University of Reading, is to provide a low cost portable system that supports a patient's condition and their recovery in hospital or at home. This is done by providing engaging applications with targeted gameplay that is individually tailored to the rehabilitation of the patient's symptoms. The applications are capable of real-time data capture and analysis in order to provide information to therapists on patient progress and to further improve the personalized care that an individual can receive.
\end{abstract}

Keywords-brain injuries; data capture; Kinect; personalised care; real-time; targeted gameplay.

\section{INTRODUCTION}

Neurological trauma causes over 250,000 hospitalizations in the UK each year, with half arising from traumatic brain injuries [1]. There are currently over one million people living with long term disabilities as a result of brain injury, and with the life expectancy of survivors being equivalent to that of the normal population, the numbers of people requiring treatment and support will continue to increase.

PURR (Prescription Software for Use in Recovery and Rehabilitation) is a system developed for use in the rehabilitation and assessment of patients following a serious brain injury. The computerized system provides engaging and medically relevant experiences tailored to an individual's requirements. These experiences are delivered through game based applications which are targeted at improving the specific symptoms experienced by an individual as a result of their injury, set within a game environment customised to their tastes and interests in order to increase their engagement in the therapy. Engagement in the recovery process is important because it encourages people to invest more time in their recovery, achieving faster and more positive results.

This project has been running in co-operation with the Royal Berkshire NHS Foundation Trust and Headway, a brain injury association and charity. It extends upon the preliminary work carried out by Professor Malcolm Sperrin and team in using the Kinect for rehabilitation [2].

\section{REHABILITATION AND ASSESSMENT METHODS}

\section{A. Current Rehabilitation Programme}

Following the incident, patients are often admitted into hospitals for immediate care and rehabilitation. The treatment received is often intense with frequent assessments made [3]. Teams of therapists provide input into the recovery of each individual and tailor care to the specific symptoms a patient is suffering. This is referred to as the acute phase.

As individuals transition from the acute phase toward recovery they may receive community and outpatient care with less direct input from medical professionals, this often involves a regime of exercises to stimulate physical and cognitive improvement.

Some patients do not make a significant recovery and are left with life-long debilitating chronic conditions. They may receive care from hospitals and charities, but this is tailored to managing the condition rather than rehabilitation with objectives of recovery.

Many current rehabilitation and assessment techniques must be facilitated by a health care professional; this reduces the number of patients that can receive concurrent care, and limits the flexibility of the processes and the extent to which it can be tailored to an individual.

As a patient improves and is discharged from the ward, the rehabilitation provided reduces, often slowing or stagnating patient progress. Often, the activities and exercises recommended by therapists are neglected due to the repetitiveness and difficulty [4].

\section{B. Improving Rehabilitation with Technology}

A number of projects have been completed which aim to improve hands-off rehabilitation using inexpensive consumer devices. In 2009 the Nintendo Wii was used as it allowed tracking of a single point and could be used for improving coordination and cognitive function [5]. However, this device is 
limited as it is unable to assess whole body movements and motor control.

The Microsoft Kinect, another consumer device released in 2010 targeted at the gaming market, is a motion sensing input device capable of tracking the whole human body. This device has been used as a rehabilitation tool due to its low cost and high flexibility [6]-[8]. The Royal Berkshire NHS Foundation Trust has also used Kinect with consumer games as a supplementary treatment, and has received a positive reception from patients. However, patients have been unable to make full use of many of the games due to their complexity and the need for more tailored therapy, problems which have also been identified in other games-based rehabilitation research [9], [10].

Off the shelf games can only be supported anecdotally, as game scores are not truly representative of improvement. The games are unable to provide usable metrics on patient recovery and therefore have limited medical benefit for healthcare professionals in assessment of patient progress. This is one of the core issues that the new system has set out to resolve.

\section{PRESCRIPTION SOFTWARE FOR USE IN RECOVERY AND REHABILITATION}

PURR is a purpose built 'prescription software' rehabilitation and assessment system for use with patients in all stages of neurological injury rehabilitation. There are two subsystems running in parallel to aid the patient in recovery, and the healthcare professionals in assessment. These are the patient rehabilitation experiences and the metrics and monitoring engines.

\section{A. Patient Rehabilitation Experiences}

The application provides a number of patient rehabilitation experiences (PREs) built to help improve upon certain characteristics of their condition. A clinician is able to configure the system and provide a plan tailored 'or prescribed' to an individual's clinical needs.

The PREs are interactive environments with objectives to be met by the patient. The patient stands in the area in front of the Kinect device (play-space) and can interact with motions and gestures to influence the environment.

Patient engagement with traditional rehabilitation exercises is often poor as activities are repetitive and have no immediate feedback. PREs are designed with gaming mechanics in mind, using many of the techniques to improve end-user engagement. The PREs can operate as mini-games, challenging the patient to perform activities normally limited by their injuries; if they are fully immersed they may focus more on the task set than their condition, inspiring them to perform better than they had previously thought possible. Feedback is given instantly for exceeding goals, which is something not possible with 'offline' exercises.

Each experience dynamically alters to the abilities of a patient. If a patient struggles to complete an action in a timely manner the next similar iteration will be simplified. This allows the application to find the level someone is at and provide challenges that scale with ability and facilitate recovery.
As the demographic of users is quite wide, ranging across ages, backgrounds and interests; the environments can be configured to be more visually appealing and engaging to a specific audience. An example of this is with the 'stop the balls' experience where the environment can be set in space or in a field; this is just an example of what is possible, new dynamic environments can be created in a very short space of time.

Currently there are three PREs, each with environment customisation. The initial experiences use gestures to identify patient's temporal and spatial responses to stimuli under certain conditions. These experiences pop characters into the screen quadrants and the patient must raise (or lower) their arm to hit the target. This tests temporal resolution, but can be also be used for cognitive training with pattern recognition etc.

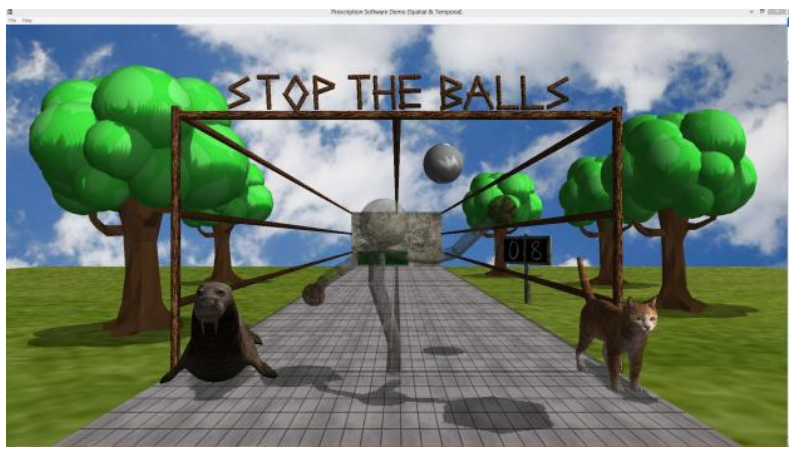

Figure 1. A PRE with a user deflecting the oncoming balls (cognitve and gross motor control). (figure caption)

One PRE that demonstrates many of the system capabilities is 'Stop the Balls' (see figure 1); the objective is to prevent any of the balls from passing the screen avatar (controlled using Kinect). As mentioned above, this can be used for testing temporal resolution and pattern recognition, but also to identify and analyse how certain movements are performed. These metrics can be used by therapists to identify improvement and target weaknesses with future tasks and exercises.

\section{B. Metrics for Healthcare Professionals}

In order for a new rehabilitation method to be deemed medically relevant it must first be proven to have an effect on patient wellbeing and recovery. Off-the-shelf packages can provide anecdotal evidence, but hard evidence can only be provided by an underlying metrics engine.

In collaboration with medical professionals, important metrics in assessing conditions have been agreed on and implemented into the system. This data can be used in real-time for dynamically altering the running of the application (e.g. targeting treatment), in real-time assessments, or for offline analysis. Physiotherapists and occupational therapists are keen to capture data autonomously whilst the patient is moving in a natural way.

The application can identify users across multiple sessions and resume their bespoke programme. The data stored can be used to monitor progression over time, helping to quantify the effects of treatment. 
The graphs in Figure 2 are indicative of improvement in response to the stimuli over a number of 'attempts', where an attempt is a successful gesture toward the stimulus. Each point has one of four colours, each corresponding to a specific screen quadrant. If there was an asymmetry in the users condition, one particular colour would form a trend separate to the others (see Figure 3). The best fitting line is plotted to show the overall rate of improvement in the session. The smaller grey line shows the improvement in the most recent subset of data (20\%). Sessions could also be compared across patients and control groups to identify atypical responses.

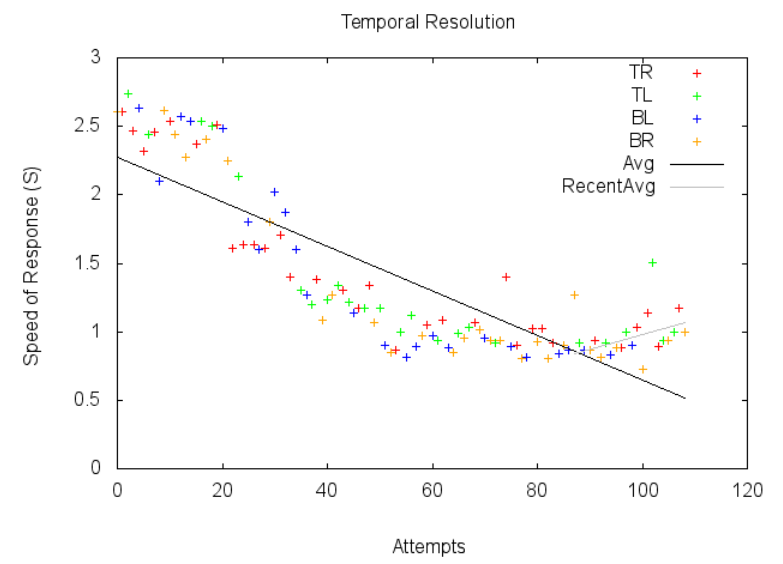

Figure 2. A real-time graph plot showing the improvement in response time.

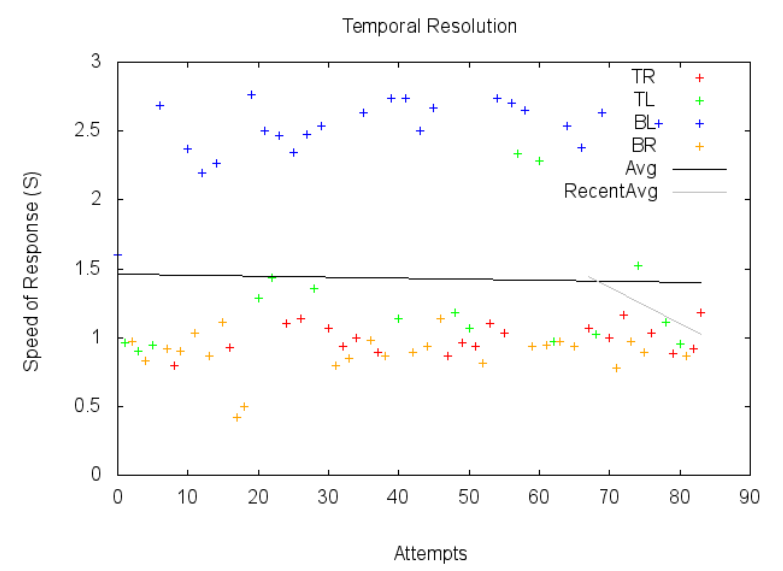

Figure 3. A real-time graph plot showing an asymmetry in response time to stimuli in the bottom left (BL) quadrant.

\section{FeEdBACK AND User Testing}

The system has been trialed with individuals shortly after hospitalization and those suffering long-term chronic conditions. Many trials have been performed with Headway, a brain injury support charity, to identify how the system works with people with serious disabilities. Many of their patients with cognitive impairments had previously resisted using Xbox titles due to the complexity involved in setting up and understanding the objectives. They have found the PREs simple to interact with and find the pace allows them to successfully interact without fear of 'failing'. They also commented on how well the PREs can adjust the pace of a game so that it can cater for an individuals' asymmetry of response.

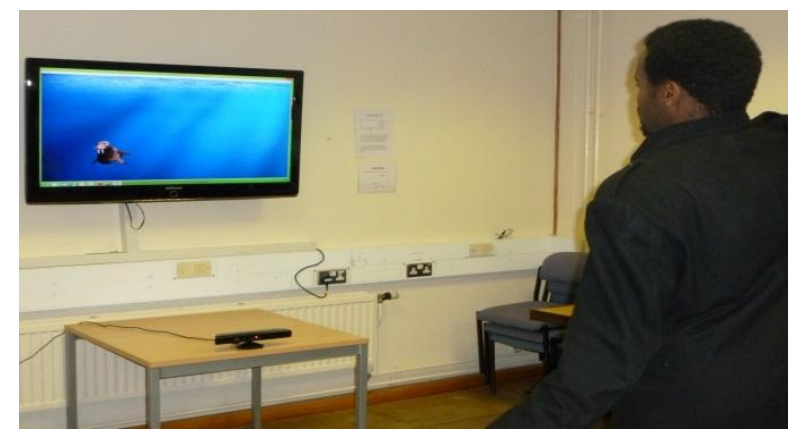

Figure 4. Early user testing at the University of Reading.

Healthcare professionals have found the information produced is very accurate and correlates strongly with offline assessments carried out by therapists. It can also provide data unobtainable through traditional methods. In particular the progression of a motion and how individuals compensate for restricted joint movements was seen in a number of cases where patients had very little rotation in their elbows. One physiotherapist commented that "the information collected by the application really helps speed up patient assessment" and "patient motivation is much better with the ball game than the exercises we recommend, getting people involved and active makes a real difference".

\section{CORE TECHNOLOGIES AND DEVELOPMENT}

The software, written in $\mathrm{C}++$ and developed using Microsoft Kinect and DirectX technologies has two-layer architecture as detailed below.

\section{A. Core Framework Layer}

The 'core' layer provides all of the underlying services to the PREs through an extensible API. The system is designed carefully to keep each service discreet and pluggable in order that it is possible to extend functionality with changes having minimal impact on other system components.

This layer provides a custom written graphics and rendering pipeline using the low level DirectX 11 APIs. This engine is capable at rendering the scene at over 60 frames per second for smooth performance. The pipeline fully supports many of the popular graphics processing techniques, including lighting, shading, normal maps, skinned mesh animation, shadows and texturing amongst many others. Complex models are created externally so there is a model import pipeline to allow the creation of scenes in 3D modeling packages such as Blender [11] or Autodesk 3DS Max [12]. A custom physics engine provides an artificial physics and collision simulation; this makes scenes reflect real world environments more realistically, which matches people's expectations when dealing with certain scenarios.

Resource management is an important consideration in realtime applications, loading on-demand can have performance issues when running on lower end hardware (the target platform). This was resolved by asynchronously loading blocks 
of resources at load time, increasing initialisation time by a few seconds but guaranteeing smoother experiences.

Kinect is the primary input device for end-users of the system; this device is used for all the medical monitoring and environment interactions. This is wrapped into a Kinect and Gestures system for interfacing with those features released by Microsoft and augmenting them with added functionality.

The metrics engine is written to support the clinicians and therapists; it collects data on the session and can be configured to store data on specific aspects of the condition. For example, medical professionals are able to select to monitor joint orientation and compile a report from the session. Some data can be visualised in real-time, such as spatial and temporal locality, with graphs and targeted metrics windows.

\section{B. Application Layer}

The 'application' layer contains all of the PRE layer technologies, including the interfaces for targeting applications to individual patients and conditions. The aim is for it to ultimately be scriptable by a non-programmer so that they can develop new PREs.

The PURR Runtime is used to execute each of the loaded PREs using the 'core' services. This distinction ensures there is no dependency between systems allowing for new functionality to be defined without compromising application integrity.

Each object in the scene (avatar, balls, walls etc.) is implemented as an object with plug-in components and behaviours. This allows any objects to be given behaviours without writing code; this could allow entire PREs to be created with no programmer involvement.

If the system were to be deployed larger scale, the 'Application Layer' would function as the Software Development Kit (SDK) whilst the core would remain concealed.

\section{FURTHER WORK}

The application has been trialed with patients following discharge from hospital and with patients suffering long term effects. The next phase of trials will be conducted with patients in the acute stage to see how this system can be used to augment treatment already offered in a hospital setting.

There is great external interest in the project from Microsoft, hospitals, therapists and patients alike. The project is now stable enough to support a wealth of new PRE developments and deployments to match the requests of all those involved. Interest has been shown in its use for monitoring and assessing a range of medical conditions in addition to brain injury rehabilitation.

\section{CONCLUSION}

The PURR system meets the objectives of improving upon rehabilitation options currently available to individuals; the PREs have had positive feedback and have been successful in early trials. Therapists are keen to learn more about this technology and how it can help them with more challenging cases. They continue to provide valuable input on key functionality. As this project has been undertaken in collaboration with the Royal Berkshire NHS Foundation Trust, there has been medical input from the very beginning of the project ensuring all work done is relevant. There are numerous routes open to further progress the project.

\section{ACKNOWLEDGMENT}

We are very grateful for the contributions made and evaluations undertaken by the staff and patients of Headway.

\section{REFERENCES}

[1] Headway, "Brain Injury Statistics," [Online]. Available: https://www.headway.org.uk/key-facts-and-statistics.aspx

[2] Microsoft, "Microsoft in Health, The "Kinect Effect"," [Online]. Available: http://www.microsoft.com/health/en-gb/articles/Pages/kinecteffect.aspx.

[3] Department of Health, "National Stroke Strategy," Department of Health. [Online]. Available http://www.nhs.uk/NHSEngland/ NSF/Pages/Nationalstrokestrategy.aspx.

[4] R. Campbell, M. Evans, M. Tucker, B. Quility, P. Dieppe and J. L. Donovan, "Understanding non-compliance with physiotherapy in patients," J. Epidemiology and Community Health, vol. 55, no. 2, pp. 132-138. 2001

[5] D. Robbins, J. Morrison and P. Bowlby, "Wii-based compared to standard of care balance and mobility rehabilitation for two individuals post-stroke," in Proc. Virtual Rehabilitation International Conference, 117 - 120 June 2009.

[6] J Broeren, J Jalminger, L- $\AA$ Johansson, A Parmerud, L Pareto, M Rydmark, "Information and communication technology - a personcentered approach to stroke care," in Proc. 9th Intl Conf. Disability, Virtual Reality \& Associated Technologies, Laval, France, 2012.

[7] Y-J. Chang, S-F. Chen, J-D. Huang, "A Kinect-based system for physical rehabilitation: a pilot study for young adults with motor disabilities," J. Research in Developmental Disabilities, vol. 32, no. 6, pp. 2566-2570, 2011

[8] B. Lange, C.Y. Chang , E. Suma, B. Newman, A.S. Rizzo, M. Bolas, "Development and evaluation of low cost game-based balance rehabilitation tool using the Microsoft Kinect sensor", In Proc. IEEE Conf Eng Med Biol Soc. 2011 pp. 2011:1831-4. 2011.

[9] R. Lloréns, S. Albiol, J.A. Gil-Gómez, M. Alcañiz, C. Colomer, E. Noé "Balance rehabilitation using custom-made Wii Balance Board exercises: clinical effectiveness and maintenance of gains in acquired brain injury population," In Proc. 9th Intl Conf. on Disability, Virtual Reality and Assoc. Technologies, pp. 175-180, Laval, France, Sept. 2012

[10] L. Omelina, B. Jansen, B. Bonnechère, S. van Sint Jan, J. Cornelis, "Serious games for physical rehabilitation: designing highly configurable and adaptable games," In Proc. 9th Intl Conf. on Disability, VirtualReality and Assoc. Technologies, pp. 195-201, Laval, France, Sept. 2012.

[11] Blender, "Blender 3-D creation," [Online]. Available: http://www.blender.org/

[12] Audodesk 3DX Max, "Modelling and rendering software," [Online]. Available:http://www.autodesk.com/products/autodesk-3dsmax/overview 